\title{
Detector Dead Time Determination and Optimal Counting Rate for a Detector Near a Spallation Source or a Subcritical Multiplying System
}

\author{
V. Bécares and J. Blázquez \\ Division of Nuclear Fission, CIEMAT, Avenida Complutense 40, 28040 Madrid, Spain \\ Correspondence should be addressed to V. Bécares, vicente.becares@ciemat.es \\ Received 14 November 2011; Revised 10 February 2012; Accepted 14 February 2012 \\ Academic Editor: Alberto Talamo
}

Copyright (C) 2012 V. Bécares and J. Blázquez. This is an open access article distributed under the Creative Commons Attribution License, which permits unrestricted use, distribution, and reproduction in any medium, provided the original work is properly cited.

The operation of accelerator-driven systems or spallation sources requires the monitoring of intense neutron fluxes, which may be billions-fold more intense than the fluxes obtained with usual radioactive sources. If a neutron detector is placed near a very intense source, it can become saturated because of detector dead time. On the contrary, if it is placed far away from the source, it will lose counting statistics. For this reason, there must exist an optimal position for placing the detector. The optimal position is defined as the one with the minimal relative uncertainty in the counting rate. In this work, we review the techniques to determine the detector dead time that can be applied with an accelerator-driven subcritical system or a spallation source. For the case of a spallation source, counting rates do not follow Poisson's statistics because of the multiplicity of the number of neutrons emitted by incident proton. It has been found a simple expression that relates the optimal counting rate with the source multiplicity and the uncertainty in the determination of the dead time.

\section{Introduction}

A strong interest in intense accelerator-driven neutron sources for different applications has grown in the last decades. Although a number of reactions is available for neutron production with accelerated particles (photoneutrons, D-Be...), preferred technology for these neutron sources is spallation, because it provides the highest neutron yield per unit of energy of the incident particles. Neutron spallation sources consist of a high-energy proton accelerator coupled to a high $\mathrm{Z}$ target. Typically, a $1000 \mathrm{MeV}$ incident proton beam could produce $\sim 50$ nucleons per incident proton, approximately half of them neutrons [1]. In this way, very intense neutron fluxes can be obtained (over $10^{15} \mathrm{n} / \mathrm{cm}^{2} \mathrm{~s}$ ). Although current mode detectors are generally preferred for monitoring such high fluxes, pulse mode detectors are also of interest because of their ability to record information from the individual detector signals. However, if a pulsed mode detector is used to monitor such high fluxes, it can become saturated because of dead time effects.
An important application of spallation sources is to drive accelerator-driven systems (ADSs). An ADS consists of a subcritical reactor driven by an external spallation neutron source. The interest in these systems has risen since the 1990s [2-6] as it has been recognized their ability to reduce the volume and radiotoxicity of high-level nuclear waste. Operation of ADSs requires the monitoring of the neutron source intensity and the neutron flux within the reactor, for instance, to apply reactivity monitoring techniques such as the current-to-flux technique or pulsed neutron source (PNS) techniques [7-9]. Again, for this purpose, it is required to measure neutron fluxes high enough to likely cause saturation of the detectors used.

Therefore, the operation of both spallation sources and ADSs requires the monitoring of intense neutron fluxes that can cause detector saturation problems. Hence, a good characterization of the detector dead times becomes mandatory.

The measurement of dead times is usually accomplished using two constant-intensity neutron sources (the so-called 
two-source method). However, in Section 2 we propose alternative techniques that are suitable to be directly applied to measurements in spallation sources or ADS, without the need of dedicated calibration experiments and that can provide a better characterization of the dead time of the detector system than the two-source method. These techniques have been applied to the experimental results obtained at the Yalina-Booster subcritical facility $[10,11]$ during the EUROTRANS [12] experimental campaign carried out in this facility.

Finally, the combination of the saturation effects at high counting rates and the poor statistics at low counting rates cause that there must be an optimal counting rate with a minimum relative uncertainty. The determination of this optimal counting rate for the case of an spallation source is complicated by the non-Poissonian nature of the spallation process. This problem will be addressed in Section 3 .

\section{Measurement of Dead Time}

The dead time of a detector is defined as the minimum time interval that two consecutive counts must be separated in order to be recorded as two different events. The effect of having a dead time in a detector used to monitor counting rates is that the measured counting rates will be lower than the real ones. However, the real counting rate can be determined from the measured one if the dead time of the detector is known.

To obtain a formula for relating the real and the measured counting rates in a detector system affected by dead time, it must be taken into account that there are two fundamental behaviors for the dead time. They are referred as paralyzable and non-paralyzable $[13,14]$ (also called extendable and nonextendable, resp.). In a detector affected by paralyzable dead time, events that occur during the dead time of a previous one, and consequently not recorded, also produce a dead time. In other words, the effect of this last event can be regarded as extending the dead time of the previous one. On the contrary, in a non-paralyzable detector, events occurred during the dead time of a previous event are neither detected nor cause an additional dead time. For instance, fission chambers are affected by paralyzable dead time because a fission occurring during the time that the detector is ionized after a previous event also causes additional ionization, thus extending the dead time. On the contrary, some elements of the electronic chains can introduce a non-paralyzable dead time if they ignore the counts arrived during the time they are processing or recording a previous one.

The relationship of the real counting rates with the measured counting rates is well known for these two basic models. If we denote by $N$ the real counting rate, by $M$ the measured counting rate, and by $\tau$ the dead time and considering that the non-dead time disturbed distribution is Poissonian, $M$ and $N$ are related by

$$
N=\frac{M}{1-M \tau}
$$

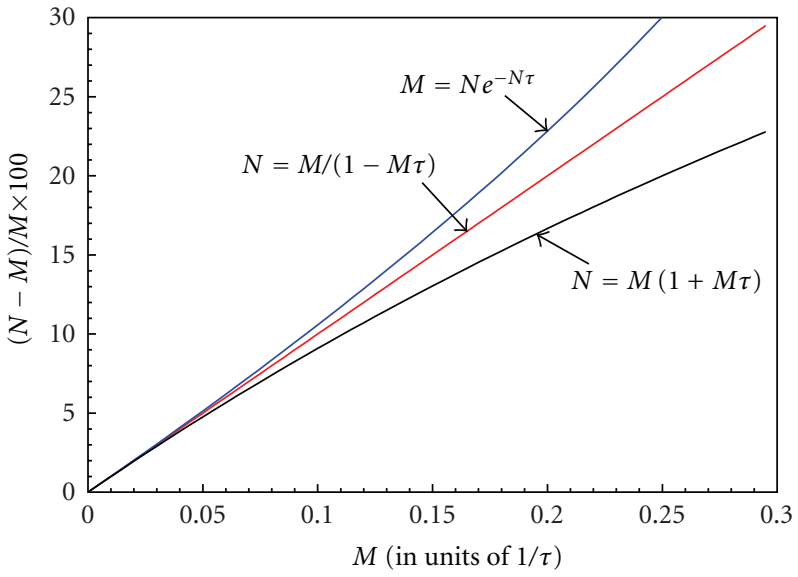

Figure 1: Relative value of the dead time correction for different counting rates considering (1), (2), and (3).

for the case of a non-paralyzable dead time, and by

$$
M=N e^{-N \tau},
$$

for the case of a paralyzable dead time.

These formulae are derived for an initially Poissonian process. This is the case of radioactive decay, but not the case of either fission or spallation. In the case of nuclear fission, the probability to have a fission in the immediate instants after a previous one is higher than that for the Poissonian process because of the secondary neutrons emitted in the fission process. In fact, the non-Poissonian nature of the fission process, constitutes the basis of the neutron noise techniques. Nevertheless, for dead time correction purposes the deviation from the Poisson distribution is small enough and the formulae derived for the Poisson process can be applied for fission. The spallation process is not a Poissonian process. This fact will be further discussed in Section 3.

In practice, however, these two models of dead time are idealized models, and actual detectors systems have neither a paralyzable nor a non-paralyzable dead time, but a combination of both. Furthermore, detector systems comprise additional elements (amplifiers, etc.) that add additional dead times to the dead time of the detector itself. Hence, it is common to study the behavior of series arrangements of dead times. Quite obviously, a series arrangement of dead times is only relevant when the first dead time is shorter than the following ones, because otherwise only the first dead time is to be taken into account. See, for instance, [14] for further details. Notice that for small dead time corrections, that is, if $\tau \ll 1 / N$, both correction formula (1) and (2) can be approximated by the same first-order Taylor term:

$$
N=M(1+M \tau) \text {. }
$$

And therefore, first-order dead time corrections are independent on the model of dead time. The relative value of the dead time correction considering each one of (1), (2), and (3) for different counting rates is presented in Figure 1.

Several techniques are available for the experimental determination of dead times $[13,14]$. The most simple one 


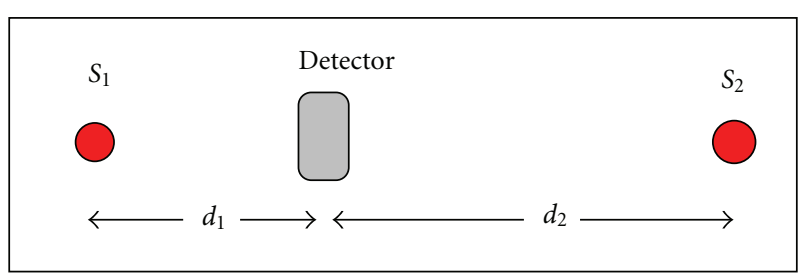

Figure 2: Two-source method.

is possibly the two-source method, described in Section 2.1. Beside these techniques, in this study we suggest another two techniques for dead time determination. The first technique (Section 2.2) is applicable with variable intensity sources such as spallation sources or ADSs. With the second technique (Section 2.3), the dead time is determined from the distribution of time intervals between consecutive detector counts.

2.1. The Two-Source Technique. The most usual technique to measure the dead time $\tau$ is the two-source technique $[13,15]$. Let us consider that we have two sources $S_{1}$ and $S_{2}$ placed at distances $d_{1}$ and $d_{2}$ from the detector, as it is shown schematically in Figure 2.

In the absence of sources, the detector gets only the background radiation level $B \mathrm{c} / \mathrm{s}$. When the source $S_{2}$ is removed and only $S_{1}$ is present, the detector gets $N_{1}+B \mathrm{c} / \mathrm{s}$ and detects $M_{1} \mathrm{c} / \mathrm{s}$. Therefore, from (3),

$$
N_{1}+B=M_{1}\left(1+M_{1} \tau\right) .
$$

Similarly, in absence of $S_{1}$ and with only $S_{2}$ present, we will have that,

$$
N_{2}+B=M_{2}\left(1+M_{2} \tau\right) .
$$

And with both sources,

$$
N_{1}+N_{2}+B=M_{12}\left(1+M_{12} \tau\right) .
$$

Working out the dead time from these three equations,

$$
\tau=\frac{M_{1}+M_{2}-M_{12}-B}{M_{12}^{2}-M_{1}^{2}-M_{2}^{2}} .
$$

From the uncertainties in the right-hand side (Poisson statistics)'s it is immediate to estimate the uncertainty in the dead time. The background $B$ has been included in case that sources are not intense enough. Usually, it can be neglected, but this simplification introduces a systematic error in the measurement.

\subsection{Dead Time Determination Varying the Source Intensity.} In an ADS or, more generally, in an accelerator-driven neutron source, the ability to change the accelerator intensity can be applied for detector dead time determination. Typically, the neutron flux measured at any point of the assembly is proportional to the source intensity. Therefore, if the detector used to measure the flux within the assembly is not affected by dead time, the measured counting rate in this detector will be linear with the accelerator intensity; say $N=k I_{A}$. If it is affected by dead time, the measured counting rate in the detector will be related with the source intensity by an equation similar to (3):

$$
k I_{A}=M(1+M \tau) \Longrightarrow \frac{I_{A}}{M}=\frac{1}{k}+\frac{\tau}{k} M .
$$

Hence, from the linear fit of $I_{A} / M$ to $M$, the dead time $\tau$ of the detector can be determined. This technique can be also applied using the counting rates in a second detector with a much lower sensitivity so that it is not affected by dead time, instead of the accelerator current $I_{A}$.

A variant of this technique using a single detector can be also applied in an ADS if the source-jerk technique is used for reactivity monitoring. The source-jerk technique [16-18] is based on the kinetic response of the neutron flux in the system to a sudden removal of the external source. In this case, the counting rate in the flux monitors experiences a fast decay due to the decay of the prompt neutrons followed by a much slower decay due to the delayed neutrons, in such a way that it can be considered that the counting rate ends in a constant level in the millisecond scale. This situation is shown in Figure 3, where the neutron level before the source removal is denoted by $N_{0}$ and the neutron level after the source removal (due to the slowly decaying delayed neutrons) is denoted by $N_{1}$.

It can be obtained in the point kinetics model that the reactivity of the reactor (in units of dollars) is related with the ratio $N_{0} / N_{1}$ by the expression

$$
\rho(\$)=1-\frac{N_{0}}{N_{1}} \Longrightarrow N_{0}=(1-\rho(\$)) N_{1} .
$$

Equation (9) constitutes the basis of the source-jerk technique. If we consider that at the counting rate $N_{0}$ the detector is affected by dead time (at therefore it measures a counting rate $M_{0}$ ) but the counting rate $N_{1}$ is low enough not to be affected by dead time, we can write an expression similar to (8) replacing $I_{A}$ by $N_{1}, M$ by $M_{0}$, and $k$ by $1-\rho(\$)$ :

$$
\frac{N_{1}}{M_{0}}=\frac{1}{1-\rho(\$)}+\frac{\tau}{1-\rho(\$)} M_{0} .
$$

Hence, if it is possible to operate the ADS with different values of the source intensity (and therefore of $N_{1}$ and $M_{0}$ ), we can use source-jerk experiments to determine both the reactivity of the assembly and the dead time of the detector. An example of the application of this technique to the experimental results in Yalina-Booster is presented in Figure 4.

However, the application of (8) or (10) for the determination of the detector dead time, either with two detectors with different sensibilities or with a single detector and the source-jerk technique, has been found to be limited in practice by a number of causes, including the presence of spatial and energy effects that make the point-kinetics model lose validity, source stability requirements before the source removal, and dynamical effects that may cause the reactivity to vary with the power level and thus with the source intensity. 


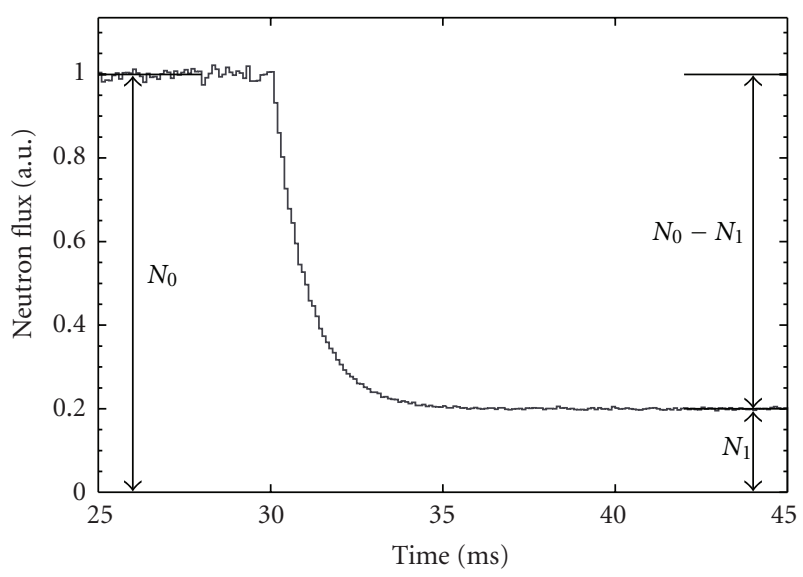

FIGURE 3: Graphical scheme of the source-jerk technique.

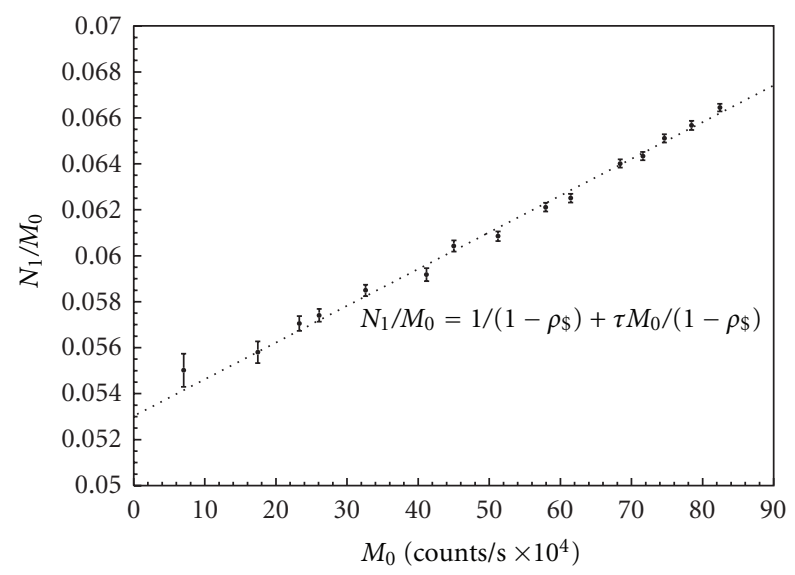

FIgURE 4: Example of the application of (10) to the source-jerk experiments at the Yalina-Booster subcritical facility.

2.3. Dead Time Determination from the Distribution of Time Intervals between Consecutive Counts. We have considered another technique to determine the detector dead time that requires a single detector and a single counting rate. This technique arises from the distribution of time intervals between consecutive counts. Modern data acquisition systems such as the one used during the EUROTRANS experiments at Yalina-Booster can register the times of individual detector signals instead of average counting rates. It is well known that for a Poissonian process the distribution of time intervals between consecutive counts (let us denote it by $\left.I_{1}(t)\right)$ takes the shape of a simple exponential. However, the presence of a dead time alters this distribution. Analytical formulae for this distribution are known for a long time for the cases of a simple paralyzable or non-paralyzable dead time [14]. For the non-paralyzable case this formula reads

$$
I_{1}(t)=N e^{-N(t-\tau)}, \quad \text { for } t>\tau ;
$$

while, for the paralyzable case

$$
I_{1}(t)=N \sum_{j=1}^{J} \frac{1}{(j-1) !}[-N(t-j \tau)]^{j-1} e^{-j N \tau},
$$

where $J$ is the largest integer below $t / \tau$. For the case of a series arrangement of dead times, the determination of the analytical shape of $I_{1}(t)$ becomes cumbersome and the reader is referred to the bibliography $[19,20]$. Nevertheless, the shape of $I_{1}(t)$ for complex arrangements of dead times can also be determined with Monte Carlo simulations. For this, we have implemented a program that generates events simulating an initial Poisson random process and then applies successively dead times of different types. Some results obtained with this program are presented in Figure 5. They are the cases of a purely paralyzable and non-paralyzable dead time, as well as the cases of series arrangements of two dead times: paralyzable/nonparalyzable and non-paralyzable/paralyzable. Normalization has been chosen so that $\int_{0}^{\infty} I_{1}(t) d t=1$ for the unperturbed Poisson distribution; with this normalization $I_{1}(0)$ is equal to the counting rate of the unperturbed Poisson distribution. Notice the clear differences in the shape of $I_{1}(t)$ among these cases. This causes that the shape of $I_{1}(t)$ can be used to determine not only the value of the dead time of the system but also its nature (paralyzable or non-paralyzable) even in the presence of complex series of dead times.

An example of the application of this technique obtained in Yalina-Booster is presented in Figure 6. The determination of the value of the dead time was straightforward, but the determination of the type of dead time or if several types were present was impossible because of the presence of some ringing effects that are visible in the figure. Notice as well that (11) and (12) are derived for a strictly constant counting rate; variations of the source intensity in actual systems also alter these results.

\section{Optimal Counting Rate with a Spallation Neutron Source}

If the detector is placed very close to a spallation neutron source, it can become saturated; if it is placed too far away, statistical resolution is very small. From here it can be inferred that there must exist an optimal position in which the relative uncertainty of the counting rate is minimal. Let us consider the case of a non-paralyzable dead time. In this case, if we denote by $m=M \Delta t$ the number of counts in the detector in the interval $\Delta t$, we have from (1) that

$$
N=\frac{m / \Delta t}{1-\tau m / \Delta t}
$$

The uncertainty in $N$ can be estimated from the variances:

$$
\sigma_{N}^{2}=\left(\frac{\partial N}{\partial m}\right)^{2} \sigma_{m}^{2}+\left(\frac{\partial N}{\partial \tau}\right)^{2} \sigma_{\tau}^{2}
$$

Partial derivatives can be computed from (13) as follows

$$
\frac{\partial N}{\partial m}=\frac{N}{m}(1+\tau N), \quad \frac{\partial N}{\partial \tau}=N^{2} .
$$

For a radioactive source, which follows a Poisson-like distribution of detection probabilities, we have that $\sigma_{m}^{2}=m$, 


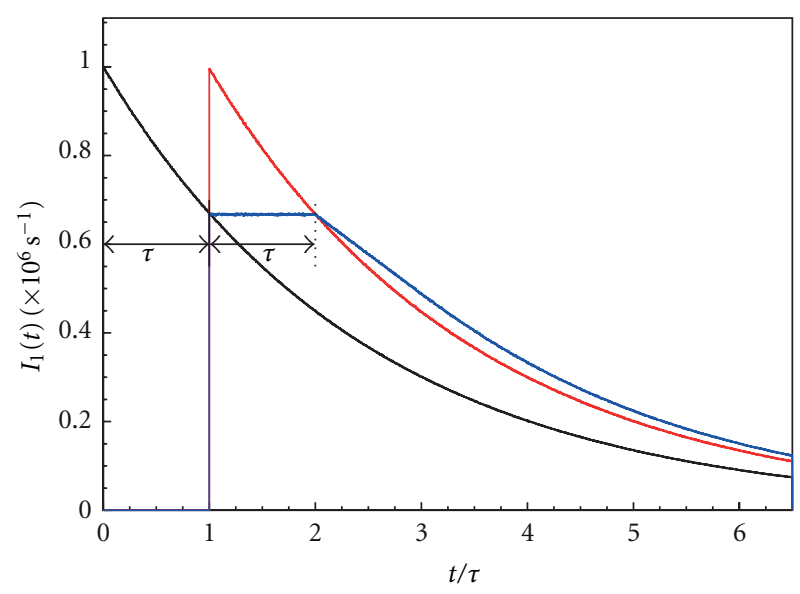

Poisson process (without dead time)

Non-paralyzable dead time

Paralyzable dead time

(a) Unperturbed distribution of time intervals and distribution of time intervals with paralyzable and non-paralyzable dead time (400 ns in any case)

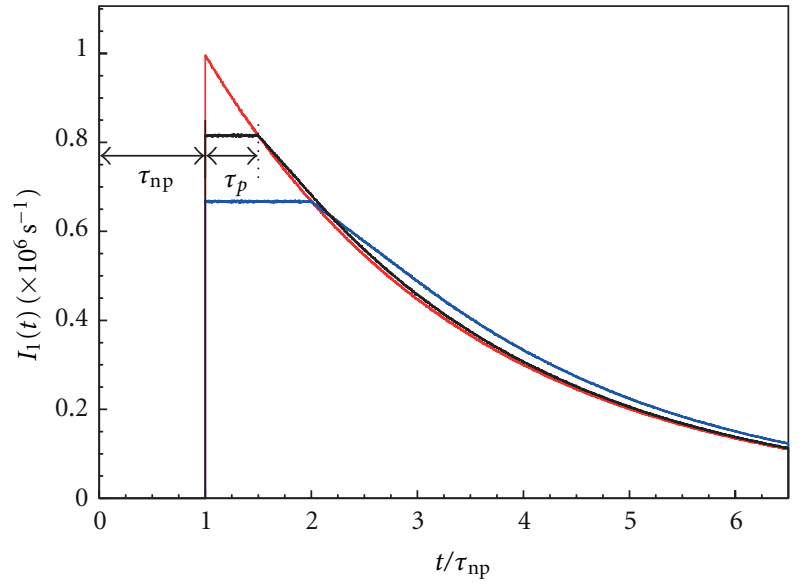

$\tau_{p}$ paralyzable $+\tau_{\mathrm{np}}$ non-paralyzable

$\tau_{\text {np }}$ non-paralyzable

$\tau_{\text {np }}$ paralyzable

(b) Distribution of time intervals for a series arrangement of a paralyzable and a non-paralyzable dead time (200 ns paralyzable and 400 ns non-paralyzable). The cases of a single paralyzable or nonparalyzable dead time (both of $400 \mathrm{~ns}$ ) are also shown for comparison

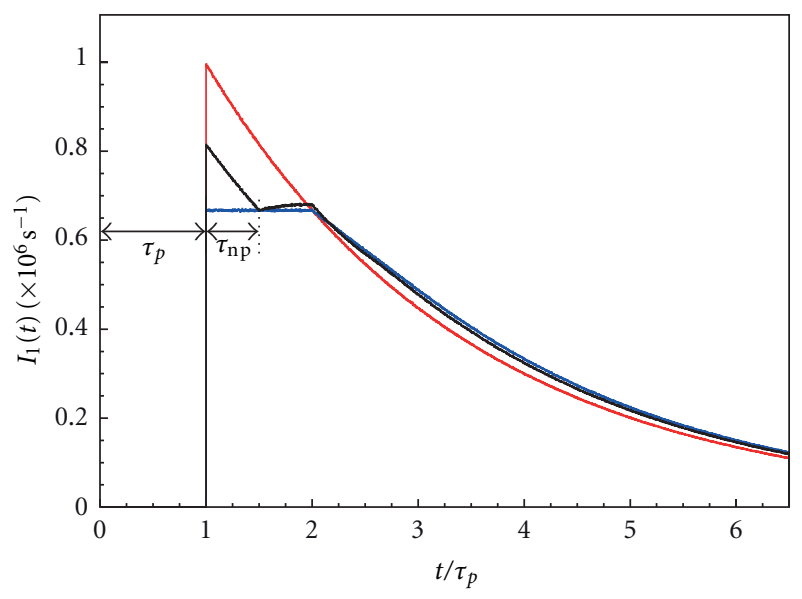

$\tau_{\mathrm{np}}$ non-paralyzable $+\tau_{p}$ paralyzable

$\tau_{p}$ non-paralyzable

$\tau_{p}$ paralyzable

(c) Distribution of time intervals for a series arrangement of a nonparalyzable and a paralyzable dead time (200 ns non-paralyzable and 400 ns paralyzable). The cases of a single paralyzable or non-paralyzable dead time (both of $400 \mathrm{~ns}$ ) are also shown for comparison

Figure 5: Distribution function $I_{1}(t)$ for intervals between adjacent random events (counting rate $10^{6}$ counts/s, $10^{9}$ counts in the simulation).

but a spallation source, because of the neutron multiplicity $\mu$, the distribution of detection probabilities differs from the Poisson distribution. In a first approximation, we have that [21]

$$
\sigma_{m}^{2}=m(1+D \mu)
$$

where $D$ is the Diven factor, which is close to the unity. Notice that if the neutron multiplicity is zero, the variance corresponds to that of a Poisson process. With these conditions,

$$
\sigma_{N}^{2}=\frac{N^{2}}{m^{2}}(1+\tau N)^{2} m(1+D \mu)+N^{4} \sigma_{\tau}^{2} .
$$

Replacing $m$ by the first-order approximation $m \simeq$ $N \Delta t(1-N \tau)$ and approximating the first term in the righthand side to the first order in $N \tau$, we find the following expression for the relative error in $\sigma_{N}$ :

$$
\varepsilon_{N}^{2}=\left(\frac{\sigma_{N}}{N}\right)^{2}=\frac{(1+3 \tau N)}{N} \frac{(1+D \mu)}{\Delta t}+N^{2} \sigma_{\tau}^{2} .
$$




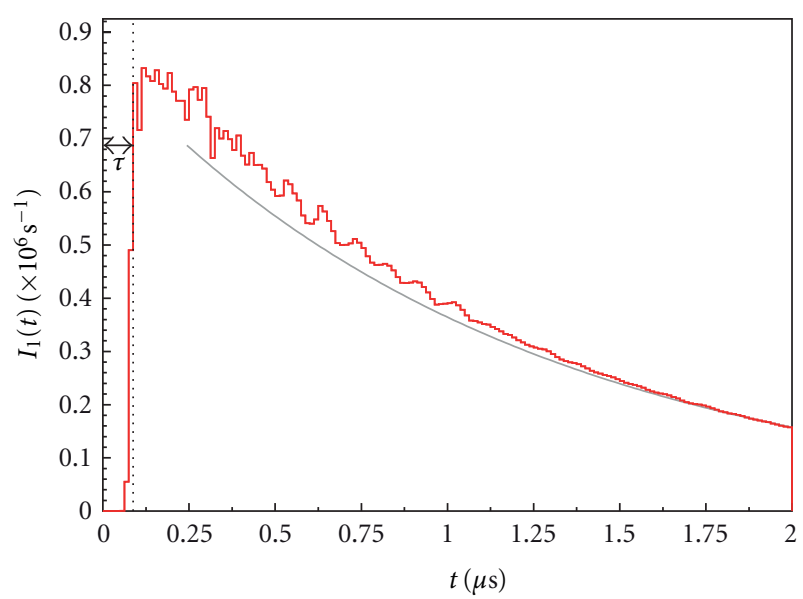

Figure 6: Example of the distribution of intervals between consecutive counts obtained from the Yalina-Booster experiments. Gray line represents the fit to a exponential (over a large range than the plotted interval).

The optimal counting rate is the one that minimizes the relative error $\varepsilon_{N}$ :

$$
\left.\frac{\partial \varepsilon_{N}^{2}}{\partial N}\right|_{N=N_{\mathrm{op}}}=0 \Longrightarrow-\frac{1}{N_{\mathrm{op}}^{2}}\left(\frac{1+D \mu}{\Delta t}\right)+2 N_{\mathrm{op}} \sigma_{\tau}^{2}=0 .
$$

Working out $N_{\text {op }}$ in this equation, we finally find that

$$
N_{\mathrm{op}}=\sqrt[3]{\frac{1+D \mu}{2 \sigma_{\tau}^{2} \Delta t}} .
$$

Typical values for the problem are given in Table 1. With these values, optimal counting rate is $1.05 \times 10^{5}(\mathrm{c} / \mathrm{s})$. Correction by dead with these parameters is about $20 \%$, in the limit of the first-order approximation (3). The only parameter one can change in (20) is $\Delta t$ so if we choose $10 \mathrm{~s}$ instead of $1 \mathrm{~s}$, the new optimal counting rate becomes $0.48 \times 10^{5}(\mathrm{c} / \mathrm{s})$, and the correction is now $10 \%$, low enough for using (3). Therefore, the detector must be placed at points with counting rates about $4 \times 10^{4}(\mathrm{c} / \mathrm{s})$. Diven's factor can be taken as the unity without a large error in the computation of the optimal counting rate.

\section{Conclusions}

In addition to traditional techniques for dead time measurement, we propose in this work two alternative methods to determine detector dead times. The first of them can be applied with variable intensity sources, such as acceleratordriven neutron sources. This method consists in studying the relationship between the detector counting rate and the source intensity that results of the presence of dead time in the detector system. A variation of this technique can be applied measuring the counting rate in a second detector with a lower counting rate (i.e., with negligible dead time effects) instead of the source intensity. Another variation of this technique, which does not require either a second detector or knowing the source intensity, can be applied in an
TABle 1: Multiplicity, Diven's factor dead time, and measurement time for a typical problem.

\begin{tabular}{lccc}
\hline$\mu$ & $D$ & $\tau(\mu \mathrm{s})$ & $\Delta t(\mathrm{~s})$ \\
\hline 20 & 1.1 & $2 \pm 0.1$ & 1 \\
\hline
\end{tabular}

ADS making most of the results of the source-jerk technique, used to determine the reactivity of the system.

The second method we propose to determine the dead time of a detector system can be applied if the data acquisition system registers the times of individual detector events instead of counting rates. In this way the distribution of time intervals between consecutive counts can be obtained, from which the detector dead time can be determined. This method has the advantage of requiring no additional detector and only one counting rate. In addition, this method is in principle capable of determining the type of dead time (paralyzable or non-paralyzable) even in the case of complex arrangements of dead times.

Finally, we remark that neutrons produced in a spallation source do not follow the Poisson statistics because of the multiplicity in the neutron production. An expression has been found that relates the multiplicity, the measurement interval, and the variance of the detector dead time. This expression allows placing neutron monitors where the relative error is minimal.

\section{Acknowledgments}

The experiments at Yalina-Booster presented in this work were supported by the IP-EUROTRANS Contract no. FI6WCT2005-516520 and the ENRESA-CIEMAT Agreement for the Transmutation Applied to High Level Radioactive Waste. The authors would like to acknowledge as well the personnel of different institutions involved at the EUROTRANS experimental campaign at Yalina-Booster, especially D. Villamarín (CIEMAT, Madrid), M. Fernández-Ordóñez (CIEMAT, Madrid) C. Berglöf (KTH, Stockholm), and finally, the support and dedication of the Yalina-Booster team at JIPNR-Sosny during the experimental campaign.

\section{References}

[1] A. Letourneau, J. Galin, F. Goldenbaum et al., "Neutron production in bombardments of thin and thick $\mathrm{W}, \mathrm{Hg}, \mathrm{Pb}$ targets by $0.4,0.8,1.2,1.8$ and $2.5 \mathrm{GeV}$ protons," Nuclear Instruments and Methods in Physics Research, Section B, vol. 170, no. 3, pp. 299-322, 2000.

[2] H. Nifenecker, S. David, J. M. Loiseaux, and A. Giorni, "Hybrid nuclear reactors," Progress in Particle and Nuclear Physics, vol. 43, pp. 683-827, 1999.

[3] A. Gandini and M. Salvatores, "The physics of subcritical multiplying systems," Journal of Nuclear Science and Technology, vol. 39, no. 6, pp. 673-686, 2002.

[4] OECD-NEA, "Accelerator-Driven Systems (ADS) and fast reactors (FR) in advanced nuclear fuel cycles. A comparative study," Tech. Rep. NEA-3109, 2002.

[5] OECD-NEA, "Physics and safety of transmutation systems-A status report," Tech. Rep. NEA-6090, 2006. 
[6] W. von Lensa, R. Nabbi, and M. Rossbach, "RED-IMPACTimpact of partitioning, transmutation and waste reduction technologies on the final nuclear waste disposal-synthesis report," in Schriften des Forschungszentrums Juelich-Reihe Energie \& Umwelt, vol. 15, 2008.

[7] P. Baeten and H. A. Abderrahim, "Reactivity monitoring in ADS, application to the Myrrha ADS project," Progress in Nuclear Energy, vol. 43, no. 1-4, pp. 413-419, 2003.

[8] E. González-Romero, D. Villamarín, M. Embid et al., "The MUSE4 pulsed Neutron source experiments," in The Physics of Fuel Cycles and Advanced Nuclear Systems-Global Developments (PHYSOR '04), pp. 1399-1407, Chicago, Ill, USA, April 2004.

[9] R. Soule, W. Assal, P. Chaussonnet et al., "Neutronic studies in support of accelerator-driven systems: the MUSE experiments in the MASURCA facility," Nuclear Science and Engineering, vol. 148, no. 1, pp. 124-152, 2004.

[10] V. Bournos et al., "YALINA-Booster benchmark specifications for the IAEA coordinated research projects on analytical and experimental benchmark analysis on accelerator driven systems, and low enriched uranium fuel utilization in accelerator driven sub-critical assembly systems," IAEA Document, 2007.

[11] H. Kiyavitskaya et al., "Experimental investigations at subcritical facilities of Joint Institute for Power and Nuclear Research-Sosny of the National Academy of Sciences of Belarus," in Technical Meeting on Use of Low Enriched Uranium in Accelerator Driven Sub_Critical Assemblies, Vienna, Austria, October 2005.

[12] European Commission, Integrated Project EUROTRANS. Annex I-Description of Work. Contract no. FI6W-CT-2004516520, 2005.

[13] G. F. Knoll, Radiation Detection and Measurement, John Wiley \& Sons, 3rd edition, 1999.

[14] International Comission on Radiation Units \& Measurements, "Particle counting in radioactivity measurements," ICRU Report 52, 1994.

[15] A. E. Profio, Experimental Reactor Physics, John Wiley \& Sons, 1976.

[16] G. R. Keepin, Physics of Nuclear Kinetics, Addison-Wesley, 1965.

[17] K. O. Ott and R. J. Neuhold, Introductory Nuclear Reactor Dynamics, American Nuclear Society, 1985.

[18] D. L. Hetrick, Dynamics of Nuclear Reactors, American Nuclear Society, 1993.

[19] H. D. Choi, "Counting statistics distorted by two dead times in series which end with an extended type dead time," Nuclear Instruments and Methods in Physics Research, Section A, vol. 599, no. 2-3, pp. 251-259, 2009.

[20] H. D. Choi, "Counting statistics modified by two dead times in series," Nuclear Engineering and Technology, vol. 43, no. 3, pp. 287-300, 2011.

[21] Y. Yamane and I. Pázsit, "Heuristic derivation of Rossi-alpha formula with delayed neutrons and correlated source," Annals of Nuclear Energy, vol. 25, no. 17, pp. 1373-1382, 1998. 

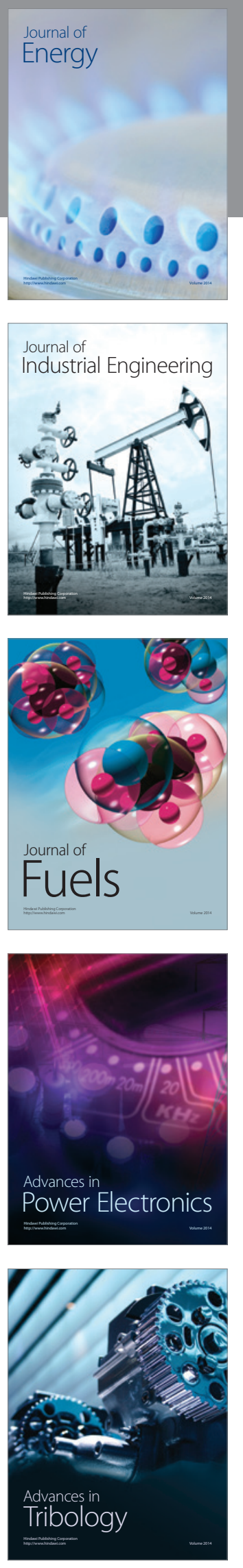
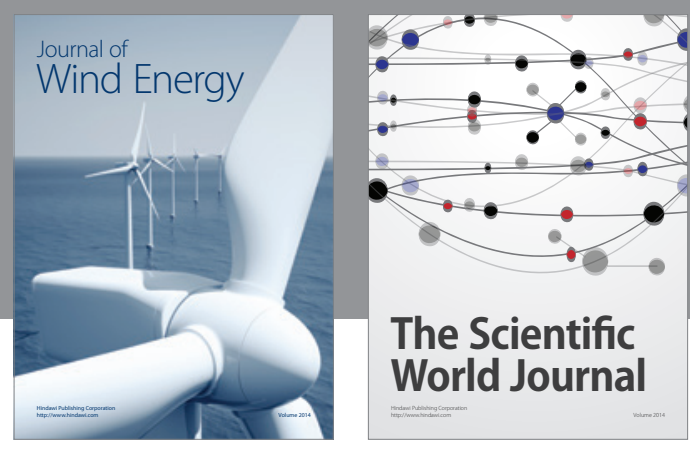

The Scientific World Journal

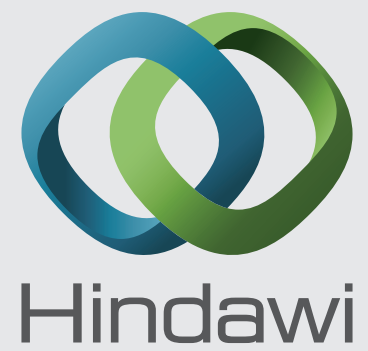

Submit your manuscripts at http://www.hindawi.com
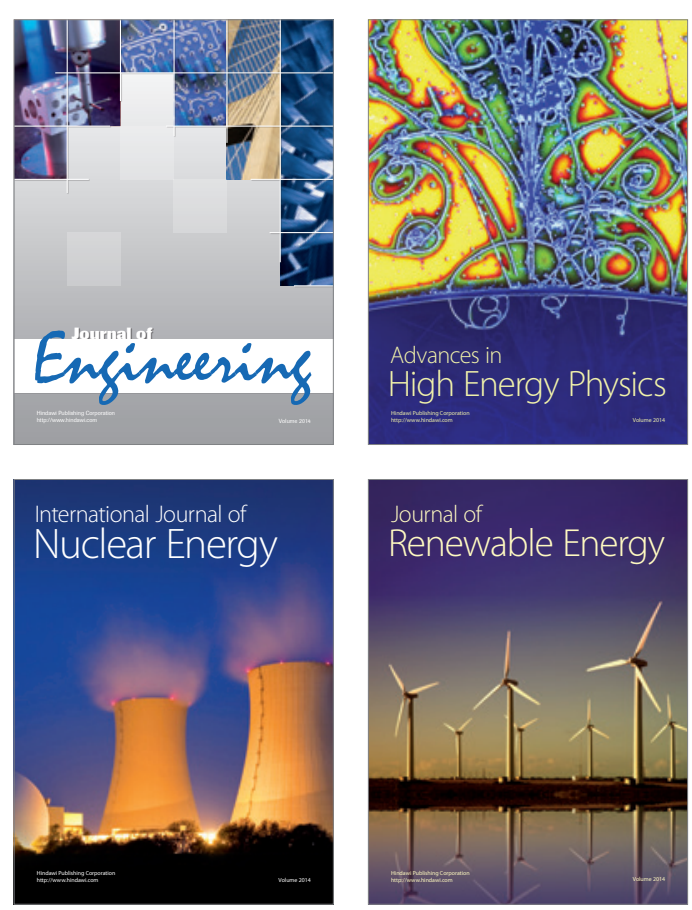

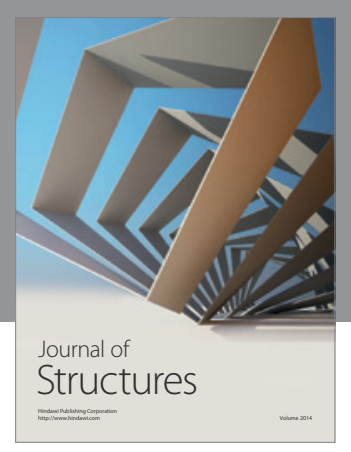

Rotating
Mechinery
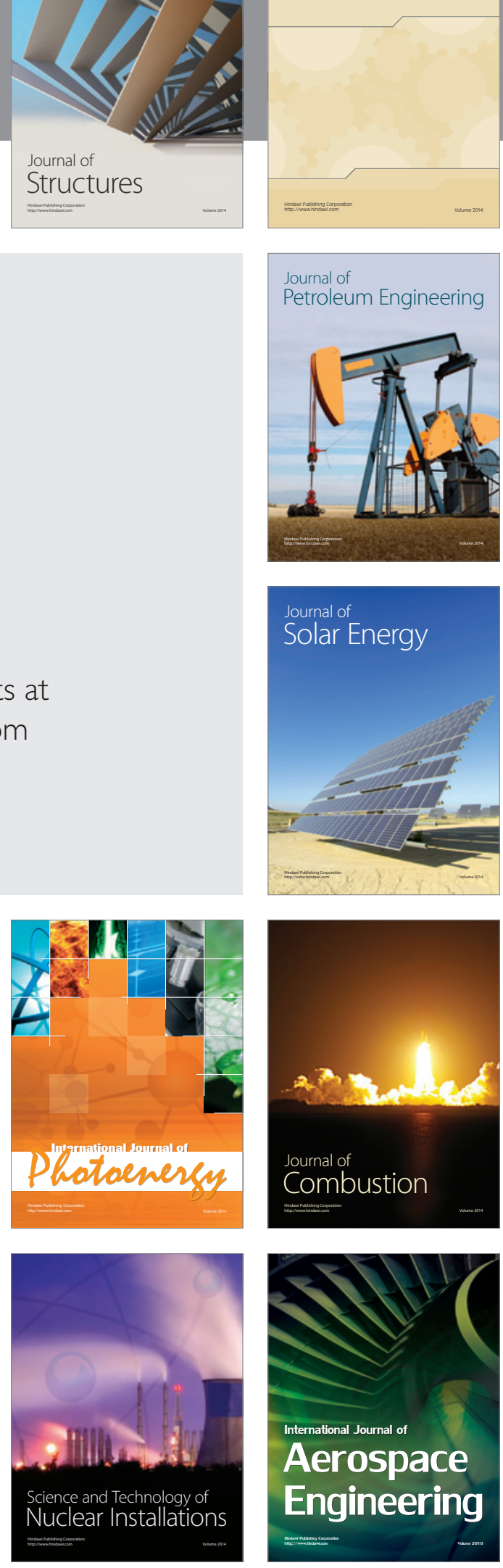